\title{
The Role of Intestinal Microbiome on Systemic Lupus Erythematosus
}

\author{
Norman Delvano Weky \\ Medical Doctor at Bhayangkara Titus Uly Hospital, Kupang, Indonesia.
}

DOI: https://doi.org/10.52403/ijrr.20220207

\begin{abstract}
Systemic lupus erythematosus (SLE) is one of the most common as chronic autoimmune disease with multiorgan target involved. The recent study found that commensal intestinal microbiome as trigger chronic autoimmunity in SLE that characterized by a significantly lower Firmicutes/Bacteroidetes ratio. It is related to activation inflammation mediators including toll-like receptors (TLRs), NOD-like receptors, adaptive immune response, and secretion of mucins antimicrobial peptides, defensins and immunoglobulin A. Other than that, lower of Firmicutes/Bacteroidetes ratio may induced activation of $\mathrm{T}$ helper cells and regulatory $\mathrm{T}$ cells, which both of these are significantly relationship in autoimmune activation for SLE. In nutrition approach for those SLE patient has been theoretically focused on the immunomodulatory effect of dietary components in the immune system activation, beside probiotic or prebiotic also significantly improve intestinal microbial related to SLE.
\end{abstract}

Keywords: intestinal microbiome, systemic lupus erythematosus, pathogenesis, autoimmune disease.

\section{INTRODUCTION}

Systemic lupus erythematosus (SLE) is one of the most common as chronic autoimmune disease with diffuse connective tissue illness that affected to multiorgan and multisystem involvement. ${ }^{[1]}$ SLE has a wide range of incidences across countries, with the global incidence in 2017 ranging above 50 to 241 per 100,000 adults. $^{[2]}$ Aside from that, $\mathrm{Mu}$ et al reported that there were 20 to 200 cases per 100,000 patients among
Asians, which affecting primarily women (but also quite common in males, children, and those over 50 years old), and targeting a variety of organs including the heart, kidney, skin, lung, and gastrointestinal tract. $^{[3,4]}$ This form of autoimmune disorder may recur and go into remission again, and typically holds of huge number of autoantibodies in the body. Apparently, the etiology of SLE is still unknown, but it has been linked to a variety of environmental (e.g., drug exposure and UV radiation), hormonal, and genetic variables.

Nonetheless, the present understanding of the relationship between intestinal microbiome and SLE has been demonstrated in a lupus-prone mouse model. ${ }^{[5]}$ The intestinal microbiome has been recommended as another crucial component. However, an insufficiency of knowledge continues to cast doubt on proofing the impact of intestinal microbiome on SLE. ${ }^{[6]}$ There are various possibilities on how the intestinal microbiome interacts with the host and how is it related with SLE, but still no definitive theories have been found. Thus, the purpose of this review is to present the current ideas and thoughts of intestinal microbiome's role on SLE.

\section{INTESTINAL MICROBIOME}

Theoretically, the human gastrointestinal system comprises of more than 100 trillion microorganisms, the most of which are found in the colon. Dysbiosis (changes in intestinal microbial population) has been linked to several autoimmune and 
chronic inflammatory disorders, including rheumatoid arthritis, type 1 diabetes, inflammatory bowel disease, and, most recently, SLE. ${ }^{[7]}$ Changes in the microbiome are influenced by a variety of factors, including gender, age, nutrition, race, stress, lifestyle (smoking habit, alcohol intake), medicine (antibiotics), and infections. ${ }^{[8]}$ According to a research by Perry et al, a number of microbiome (such as Clostridium clusters XIVa \& IVa, Lactobacillus, Eubacterium, Bacteroidetes, Firmicutes, and Bifidobacterium) are assumed to be connected to gastrointestinal physiology. ${ }^{[9]}$ In the human body system, the intestinal microbiome plays a role in immune activation. When the intestinal microbiome is out of balance, it can stimulate innate immune responses including toll-like receptors (TLRs), NOD-like receptors, adaptive immunological responses, and the release of mucins, antimicrobial peptides, defensins, and immunoglobulin A ( $\operatorname{IgA})$, which can help the immune system mature. ${ }^{[10]}$ The intestinal microbiome also had a role in the regulation and development of Peyer patches, germinal centers, and mesenteric lymph nodes (MLN), according to a research by Kamada et al. Aside from that, the intestinal microbiome controls the growth of effector $\mathrm{T}$ cells and the production of cytokines that impact the $\mathrm{T}$ helper (Th17) cells and regulatory $\mathrm{T}$ (Treg) cells, all of which have a key role in autoimmune activation in SLE. ${ }^{[11]}$

\section{INTESTINAL MICROBIOME IN SLE}

Systemic lupus erythematosus has been known as a systemic autoimmune disease characterized by the development of hyperactive and aberrant autoantibodies against nuclear and cytoplasmic antigens, as well as the activation of auto reactive inflammatory $\mathrm{T}$ cells and atypical proinflammatory cytokine release. ${ }^{[12]}$ Wei et al stated that, the intestinal microbiome may play a critical role in SLE patients which can be seen by a much reduced Firmicutes/Bacteroidetes ratio. ${ }^{[13]} \mathrm{Ma}$ et al also proposed that the commensal intestinal microbiome may be a trigger for chronic autoimmunity in SLE patients. They also discovered that the feces of lupus mice can promote the development of anti-dsDNA antibodies and an inflammatory immunological response in recipient germfree mice, which would upregulate the expression of SLE susceptibility genes. ${ }^{[14]}$

Additionally, the relative abundance of Firmicutes is lower (Fig.1c) in SLE patients, while that of Bacteroidetes is higher, indicating a lower ratio of Firmicutes to Bacteroidetes than in healthy people. [15] The overgrowth of Ruminococcus gnavus (RG) in the intestinal microbiome of a few female SLE patients differing in disease activity was initially described by Azzouz et al. RG expansions in the intestinal reflected the severity of disease activity and were common in SLE patients. ${ }^{[16]}$ According to $\mathrm{Mu}$ et al, increased intestinal permeability (leaky intestinal) and intestinal microbial product translocation have been shown to favor lupus formation and disease progression. ${ }^{[12]}$ Increased intestinal permeability and $L$. reuteri translocation into inner organs were found in TLR7-transgenic spontaneous lupus mice (Fig. 1a). ${ }^{[17]}$ As a result, Enterococcus gallinarum has been found in the mesenteric vein, intestinal-draining lymph nodes, livers, and spleens (Fig. 1b). ${ }^{[18]}$ The ileal barrier was similarly compromised in E. gallinarum monocolonized mice, as demonstrated by downregulated mucus layer and antimicrobial defense markers. ${ }^{[18]}$ Both species have the ability to activate pDCs and secrete interferon (IFN- $\alpha$ ), which are major factors to SLE pathogenesis (Fig. 1a, d). Even if the SLE patient does not have any gastrointestinal symptoms, an impaired intestinal barrier is found in SLE patients. ${ }^{[19]}$ The high quantity of serum lipopolysaccharide (LPS), a cell wall component of gram-negative bacteria, which may be eliminated by antibiotics (e.g. vancomycin) therapy, together with the relief of lupus-like manifestations in MRL/lpr mice, may imply enhanced intestinal permeability in SLE patients. ${ }^{[20]}$ 
Moreover, the plasma LPS level in SLE patients is strongly linked with antidsDNA antibody titers in the blood. ${ }^{[21]}$ Other indicators linked with intestinal barrier insufficiency, such as fecal calprotectin levels, serum soluble CD14a, and serum 1-acid glycoprotein, have also been found to be elevated in SLE patients. ${ }^{[16]}$ Lopez et al discovered that SLE patients have decreased levels of the Firmicutes to Bacteroidetes ratio (Synergistetes). ${ }^{[22]}$ Consequently, Synergistetes has the ability to lower blood levels of interleukin (IL-6) (a proinflammatory cytokine) and may induce B1 cells to generate natural protective IgM anti-phosphorylcholine under physiological settings. This can be accomplished in a number of ways [clearing of apoptotic cells and cellular debris, elimination of oxidized lipids, inhibition of mitogen-activated protein kinase (MAPK) activation, and other proinflammatory mediators]. ${ }^{[23]}$ Aside from that, patients with SLE had a lower abundance of Lactobacillaceae and a higher richness of Lachnospiraceae. ${ }^{[12]}$

Lactobacillus spp. and Lactobacillus reuteri have recently been shown to improve kidney function in rats suffering from lupus nephritis. Treatment with Lactobacillus spp. repaired intestinal permeability (altered before the beginning of nephritis), lowered inflammatory cytokines (i.e. IL-6 and IL18), and boosted anti-inflammatory cytokines (i.e. IL-10, Transforming growth factor (TGF-)) and Tregs, according to the researchers. It also showed a reduction in IgG2a (one of the primary immunological deposits) and IFN- levels, indicating that renal impairment was improving. However, no such evidence was seen in male mice, indicating that the microbiome's effect is sexually linked and dependent, and implying a role for sex hormones in the intestinal microbiome's regulatory function on lupus. ${ }^{[3,12]}$

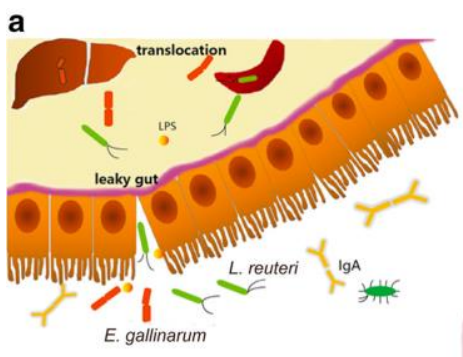

c

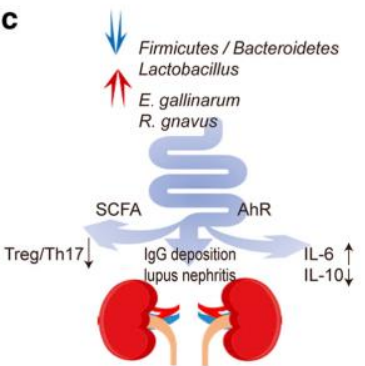

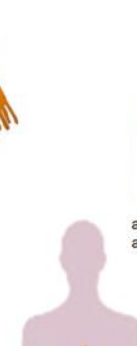

b

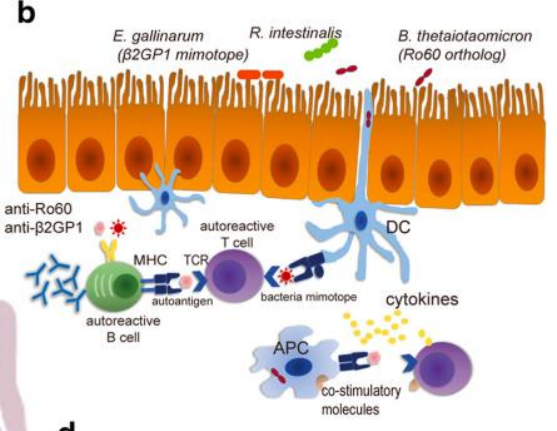

d

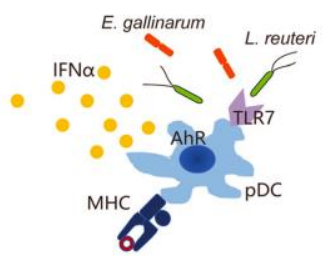

Figure 1. The pathogenesis of SLE from intestinal microbial. ${ }^{[12]}$ (a \& c) SLE patients have a limited microbial diversity in their stomach, with the possibility of expanding their microbiome. (a) Microbiome can be translocated to MLNs and the liver when intestinal permeability is compromised. (b) E. gallinarum delivers ligands to the aryl hydrocarbon receptor (AhR), and (d) Proliferation of Th17 and Tfh cells is stimulated by activation of the AhR pathway, which leads to the generation of systemic autoantibodies. E. gallinarum causes plasmacytoid dendritic cells (pDCs) and hepatocytes to produce type I interferon (IFN). TLR7dependent $L$. reuteri translocation increases the amount of pDCs and type I IFN expression, inducing SLE. Cross-reactive T and B cell responses are triggered by bacterial orthologs' molecular mimicry of human autoantigens, causing SLE.

\section{FUTURE INTERVENTION IN SLE}

\section{A. Diet intervention}

The immunomodulatory influence of dietary components in immune system activation has been conceptually highlighted in the nutrition strategy for patients with SLE. Although little is known about the influence of diet on microbiome in SLE 
patients, there is some nutrition research that suggests that part of the dietary impact on SLE pathogenesis might be done by intestinal microbiome manipulation. ${ }^{[24]}$ Regrettably, there isn't enough evidence to say if a dietary intervention can help prevent SLE recurrences. A few studies from other diseases with dysbiosis have shown the function of many dietary components in balancing particular intestinal microbiome quantities, which might be applied to SLE.

Ley et al, found a low-fat and lowcarbohydrate diet can replace the intestinal microbiome balance by boosting the number of Bacteroidetes in SLE patients with high Firmicutes/Bacteroidetes ratios. ${ }^{[25]}$ Animal proteins and saturated fats in the diet have also been linked to a higher abundance of Bacteroidetes, according to Yatsunenko et al. ${ }^{[26]}$ High consumption of whole grains, on the other hand, has been linked to a positive rise in Firmicutes/Bacteroidetes ratio in the gastrointestinal tract, and the administration of a low-fat/high-fiber diet has been linked to a positive increase in Firmicutes quantity. ${ }^{[27,28]}$ De Filippo et al discovered that long-term intake of diets rich in whole grains, dietary fibers, and vegetables resulted in decreased levels of Bacteroidetes in African children, demonstrating the health advantages of whole grains through microbiome modification. ${ }^{[29]}$ Of fact, there is no other study in the literature that specifically evaluates the impact of the amount and type of lipids in the diet on the microbiome of people with SLE. Cuervo et al found no evidence of a link between lipids or saturate fatty acids in moderate levels in a well-balanced diet and fecal microbiome in an SLE cohort in a descriptive investigation. ${ }^{[30]}$

In a previous study conducted recently, researchers found a link between fruit consumption, such as oranges and apples, and reductions in specified microbe populations in SLE patients. ${ }^{[30]}$ It's crucial to remember that these fruits are natural sources of polyphenols and fibers with a significant potential for microbiome modulation. Bifidobacteria degrades particular phenolic chemicals, such as dihydrochalcones from apples, as well as dietary fiber, encouraging its proliferation. Given the immunomodulatory impact attributed to several strains of the genus Bifidobacterium, these types of alterations might be particularly interesting for SLE patients. ${ }^{[31]}$ The Bifidobacterium bifidum LMG13195 strain, for example, increased the proliferation of Treg cells, a type of Tcell that promotes mucosal homeostasis. ${ }^{[32]}$ Another study discovered a correlation between dietary flavones and the levels of Blautia sp., a Clostridium cluster XIVA member. Because this microbiome is also involved in Treg cell development, boosting its growth through nutrition may be beneficial in restoring or maintaining immunological activation systems. ${ }^{[33]}$

Intriguingly, in animal models, a dietary therapy with vitamin A supplementation has demonstrated benefits in SLE pathogenesis, particularly in the decrease of both proteinuria and glomerulonephritis, and has been found to lessen symptoms generally. ${ }^{[34]}$ Another dietary intervention research found that retinoic acid (the active form of vitamin A retinol) supplementation restored decreased lactobacilli levels that had fallen during the development of SLE in an animal model. ${ }^{[30]}$

\section{B. Probiotics or prebiotics}

Surprisingly, taking probiotics or prebiotics has been shown to increase intestinal microbial health in people with SLE. Another study found that long-term probiotic consumption may lower lupus severity by regulating inflammatory responses and reducing autoantibody synthesis. ${ }^{[35]}$ When compared to microbiome isolated from healthy controls, microbiome isolated from SLE patient feces samples boosted lymphocyte activation and naïve CD4 + lymphocyte differentiation towards the Th17 subset, based on a study by López et al. Furthermore, when the same proportions of Bifidobacterium bifidum LMG13195 $(\mathrm{Bb})$ or a mix of two Clostridia 
strains (CI:Ruminococcus obeum DSM 25238 and Blautia coccoides DSM935) substituted 5,10 , or $30 \%$ of SLE intestinal microbiome, it was found that $\mathrm{Bb}$ and $\mathrm{CI}$ notably reduced CD4+ lymphocyte overactivation and Th17/Th1 balance. Both BB and CI are well-known for their Treg inducing effects. ${ }^{[32]}$ Mike et al found that feeding mice a Lactobacillus casei (LC) diet from the time they were weaned might extend their lives and inhibit the growth of B220+ $\mathrm{T}$ lymphocytes in the spleen and mesenteric lymph nodes. In addition, intraperitoneal injection of LC into mice dramatically reduced splenocyte proliferation and the B220+CD4-CD8-T cells accumulation, while boosting I-Amacrophages and macrophage colonyforming cells (M-CFCs). ${ }^{[36]}$

Overall, while there is a paucity of data on SLE therapy based on intestinal microbiome strategy, the present evidence leads to a feasible SLE treatment method. However, the underlying processes causing the beneficial effects of a particular diet, prebiotics, or probiotics on SLE are yet unknown, and further comprehensive study is needed. ${ }^{[37]}$

\section{CONCLUSION}

In this review, concluded that, alteration of intestinal microbiome imbalance can induce the intestinal barrier problem and microbial translocation, the consequent immune dysregulations presented as IFN pathway overactivation as well as Th17/Treg imbalance, these condition as related to SLE pathophysiology by inducing self-tolerance breakdown and autoantibody. Individualized intestinal microbiome modification such as dietary intervention, and probiotic or prebiotic supplementation may be promising intervention strategies for future SLE patient. However, future study needs for evaluate the long term of nutrition approach impact for SLE patient.

Acknowledgement: None

\section{Conflict of Interest: None}

\section{Source of Funding: None}

\section{REFERENCES}

1. Schulz SW, Derk CT. The gastrointestinal manifestations of systemic lupus erythematosus: a survey of the literature. The Open Autoimmunity Journal. 2009; 1:10-26.

2. Rees F, Doherty M, Grainge MJ, Lanyon P, Zhang W. The worldwide incidence and prevalence of systemic lupus erythematosus: a systematic review of epidemiological studies. Rheumatology (Oxford). 2017;56 (11):1945-61.

3. Mu Q, Zhang $\mathrm{H}$, Liao $\mathrm{X}$, Lin $\mathrm{K}$, Liu $\mathrm{H}$, Edwards MR, et al. Control of lupus nephritis by changes of gut microbiota. Microbiome 2017;5(73):1-12

4. Han S, Zhuang H, Shumyak S, Yang L, Reeves WH. Mechanisms of autoantibody production in systemic lupus erythematosus. FrontImmunol. 2015;6:228.

5. Bandre A, Subramanian S, Mahajan S. Role of gut microbiota in autoimmune diseases: a review. J Vaccines Immunol. 2021;6 (163):1-14.

6. Mahajan P, Kulkarni A, Bandre A, Subramanian S, Mahajan S. Role of gut microbiota in autoimmune diseases: a review. J Vaccines Immunol. 2021;6(163):1-14.

7. Hevia A, Lopez P, Suarez A, Jacquot C, Urdaci MC, Margolles A, et al. Association of levels of antibodies from patients with inflammatory bowel disease with extracellular proteins of food and probiotic bacteria. Biomed Res Int. 2014:1-8.

8. Neuman H, Koren O. The gut microbiota: a possible factor influencing systemic lupus erythematosus. Curr Opin Rheumatol. 2017;29:374-77.

9. Perry M, Swain S, Kemmis-Betty S, Cooper P. Guideline development group. multiple sclerosis: summary of NICE guidance. BMJ. 2014;349:5701.

10. Hollister EB, Gao C, Versalovic J. Compositional and functional features of the gastrointestinal microbiome and their effects on human health. Gastroenterology. 2014; 146:1449-58.

11. Kamada N, Núnez G. Regulation of the immune system by the resident intestinal 
bacteria. Gastroenterology. 2014;146:147788.

12. Li R, Meng X, Chen B, Zhao L, Zhang X. Gut microbiota in lupus: a butterfly effect?. Current Rheumatology Reports. 2021;23(27):1-9.

13. Wei F, Xu H, Yan C, Rong C, Liu B, Zhou $H$. Changes of intestinal flora in patients with systemic lupus erythematosus in northeast China. PLoS ONE. 2019;14(3):111.

14. Ma Y, Xu X, Li M, Cai J, Wei Q, Niu H. Gut microbiota promote the inflammatory response in the pathogenesis of systemic lupus erythematosus. Molecular Medicine. 2019;25(35):1-16.

15. Hevia A, Milani C, Lopez P, Cuervo A, Arboleya S, Duranti $S$, et al. Intestinal dysbiosis associated with systemic lupus erythematosus. Microbiology. 2014;5: e01548-14.

16. Azzouz D, Omarbekova A, Heguy A, Schwudke D, Gisch N, Rovin BH, et al. Lupus nephritis is linked to disease-activity associated expansions and immunity to a gut commensal. Ann. Rheum. Dis. 2019;78: 947-56.

17. Zegarra-Ruiz DF, El Beidaq A, Iniguez AJ, Lubrano Di Ricco M, Manfredo Vieira S, Ruff WE, et al. A diet-sensitive commensal Lactobacillus strain mediates TLR7dependent systemic autoimmunity. Cell Host Microbe. 2019;25(1):113-27

18. Manfredo VS, Hiltensperger M, Kumar V, Zegarra-Ruiz D, Dehner C, Khan N, et al. Translocation of a gut pathobiont drives autoimmunity in mice and humans. Science. 2018;359(6380):1156-61

19. Thim-Uam A, Surawut S, Issara-Amphorn $\mathrm{J}$, Jaroonwitchawan $\mathrm{T}$, Hiengrach $\mathrm{P}$, Chatthanathon $\mathrm{P}$, et al. Leaky-gut enhanced lupus progression in the Fc gamma receptor$\mathrm{IIb}$ deficient and pristane induced mouse models of lupus. Sci Rep. 2020;10(1):777

20. Mu Q, Tavella VJ, Kirby JL, Cecere TE, Chung M, Lee J, et al. Antibiotics ameliorate lupus-like symptoms in mice. Sci Rep. 2017;7(1):13675.

21. Ogunrinde E, Zhou Z, Luo Z, Alekseyenko A, Li QZ, Macedo D, et al. A link between plasma microbial translocation, microbiome, and autoantibody development in first-degree relatives of systemic lupus erythematosus patients. Arthritis Rheumatol. 2019;71(11): 1858-68.
22. Lopez P, de Paz B, Rodriguez-Carrio J, Hevia A, Sanchez B, Margolles A, et al. Th17 responses and natural IgM antibodies are related to gut microbiota composition in systemic lupus erythematosus patients. Sci Rep. 2016;6:24072.

23. Gronwall C, Chen Y, Vas J, Khanna S, Thiel S, Corr M, et al. MAPK phosphatase1 is required for regulatory natural autoantibody-mediated inhibition of TLR responses. Proc Natl Acad Sci USA. 2012; 109:19745-50.

24. Vieira SM, Pagovich OE, Kriegel MA. Diet, microbiota and autoimmune diseases. Lupus. 2014;23:518-26.

25. Ley RE, Turnbaugh PJ, Klein S, Gordon JI. Microbial ecology - human gut microbes associated with obesity. Nature. 2006;444: 1022-3.

26. Yatsunenko T, Rey FE, Manary MJ, Trehan I, Dominguez-Bello MG, Contreras M, et al. Human gut microbiome viewed across age and geography. Nature. 2012;486:222-7.

27. Martínez I, Lattimer JM, Hubach KL, Case JA, Yang J, Weber CG, et al. Gut microbiome composition is linked to whole grain-induced immunological improvements. ISME J. 2013;7:269-80.

28. Wu GD, Chen J, Hoffmann C, Bittinger K, Chen YY, Keilbaugh SA, et al. Linking long-term dietary patterns with gut microbial enterotypes. Science. 2011;334: 105-8.

29. De Filippo C, Cavalieri D, Di Paola M, Ramazzotti M, Poullet JB, Massart S, et al. Impact of diet in shaping gut microbiota revealed by a comparative study in children from Europe and rural Africa. Proc Natl Acad Sci. 2010;107:14691-6.

30. Cuervo A, Hevia A, Lopez P, Suarez A, Sanchez B, Margolles A, et al. Association of polyphenols from oranges and apples with specific intestinal microorganisms in systemic lupus erythematosus patients. Nutrients.2015;7:1301-17.

31. Konieczna P, Akdis CA, Quigley EM, Shanahan F, O'Mahony L. Portrait of an immunoregulatory Bifidobacterium. Gut Microbes. 2012;3:261-6.

32. Lopez P, Gonzalez-Rodriguez I, Gueimonde M, Margolles A, Suarez A. Immune response to Bifidobacterium bifidum strains support Treg/Th17 plasticity. PLoS One. 2011;6:e24776 
33. Atarashi K, Tanoue T, Shima T, Imaoka A, Kuwahara T, Momose Y, et al. Induction of colonic regulatory $\mathrm{T}$ cells by indigenous Clostridium species. Science. 2011;331: 337-41

34. Hsieh CC, Lin BF. Dietary factors regulate cytokines in murine models of systemic lupus erythematosus. Autoimmun Rev. 2011; 11:22-7.

35. Esmaeili SA, Mahmoudi M, Momtazi AA, Sahebkar A, Doulabi H, Rastin M. Tolerogenic probiotics: potential immunoregulators in systemic lupus erythematosus. J Cell Physiol. 2017;232(8): 1994-2007.

36. Mike A, Nagaoka N, Tagami Y, Miyashita M, Shimada S, Uchida K, et al. Prevention of B220+ $\mathrm{T}$ cell expansion and prolongation of lifespan inducedby Lactobacillus Casei in MRL/Lpr mice. Clin Exp Immunol. 1999;117(2):368-75.

37. Pigneur B, Ruemmele FM. Nutritional interventions for the treatment of IBD: current evidence and controversies. Therap Adv Gastroenterol. 2019;12: 1756284819890534. Published 2019 Nov 25. doi: $10.1177 / 1756284819890534$

How to cite this article: Norman Delvano Weky. The role of intestinal microbiome on systemic lupus erythematosus. International Journal of Research and Review. 2022; 9(2): 33-39. DOI: https://doi.org/10.52403/ijrr.20220207 